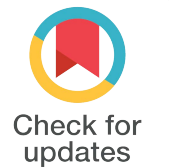

updates

DOI: $10.29252 / \mathrm{ijcp}-26694$

\section{Different Polymorphisms of Placental Growth Factor (PLGF) Gene in Iranian Women's Population with Pre-eclampsia}

\author{
Parisa Pourroostaei Ardakani ${ }^{1}$, Arezo Ramezani ${ }^{1}$, Zeinab Piravar ${ }^{1, *}$, \\ Nastaran Asgharimoghaddam ${ }^{1}$, Roudabeh Behzadi ${ }^{1}$, Mehrdad Jafari \\ Fesharaki $^{2}$ \\ ${ }^{1}$ Department of Biology, Faculty of Sciences, Central Tehran Branch, Islamic Azad \\ University, Tehran, Iran \\ ${ }^{2}$ School of Medicine, Shahid Beheshti University of Medical Sciences, Tehran, Iran \\ * Corresponding author: Zeinab Piravar, Department of Biology, Faculty of Sciences, \\ Central Tehran Branch, Islamic Azad University, Tehran, Iran. E-mail: \\ saba.piravar@gmail.com, Tel:+98-2144600183
}

\begin{tabular}{l}
\hline Submitted: 10-08-2019 \\
Accepted: 05-11-2019 \\
\hline Keywords: \\
Polymorphism, Genetic \\
Placenta Growth Factor \\
Pre-Eclampsia \\
\hline C) 2020. International Journal \\
of Cardiovascular Practice.
\end{tabular}

\section{INTRODUCTION}

Preeclampsia is a common and serious hypertensive disorder affecting approximately $5 \%$ of pregnancies. The biology of the disease is complex and not understood [1]. This disease associated with increased blood pressure more than $140 / 90 \mathrm{mmHg}$ in the second half of pregnancy and proteinuria (protein excretion in the urine) more than $300 \mathrm{mg} / 24 \mathrm{~h}$ and is considered as one of the three leading causes of maternal and fetal mortality and related complications [2]. This complication is a systemic disorder and can lead to difficulties in the mother, such as kidney and liver dysfunction, cerebral edema associated with seizure and affliction to HELLP syndrome (hemolysis elevated liver enzymes and low platelet count). As well as, the risk of injury in the fetus, such as fetal growth restriction, which is considered as one of the most important causes of neonatal mortality, is common $[3,4]$. Expulsion of the fetus and placenta from the mother's body eliminates 
the symptoms of the disease. Still, the complications of the disease can be problematic for the child and mother until the end of life. Among these are the risks of afflicting mother to cardiovascular and metabolic diseases [5]. There are two types of pre-eclampsia: mild and severe. The severe pre-eclampsia type is characterized by a diastolic blood pressure of $110 \mathrm{~mm}$ $\mathrm{Hg}$ or more, higher proteinuria, high creatinine, increased liver enzymes and headache, oliguria, pulmonary edema, upper abdominal pain, visual impairment, and thrombocytopenia [6]. Various factors are involved in the onset of this disease including Vascular activating proteins, oxidative stress, endothelial damage, immunogenic disorders, thrombophilia, and other cases $[5,6]$. Women with a history of preeclampsia have approximately double the risk for subsequent ischemic heart disease, stroke and venous thromboembolic events over the 5 to 10 years following the pregnancy $[7,8]$.

Although most cases of preeclampsia are sporadic, genetic factors are thought to play a role in disease susceptibility. More than 70 candidate preeclampsiarelated genes have been studied. Placental Growth Factor (PlGF) is essential member of the VEGF (Vascular endothelial growth factor), family with a molecular weight of $75.13 \mathrm{~kb}$. PlGF was proposed to regulate vascular development and trophoblast growth and differentiation. PlGF is responsible for placentation, and it is believed to play its role as antigenic [7]. Unlike VEGF which is required for angiogenesis and endothelial cell maintenance, PIGF is redundant for vascular development and selectively binds Flt-1/ sFlt-1 (soluble FMS-like tyrosin kinase-I). PlGF is necessary for stimulating endothelial cell growth, migration, and survival and plays a primary role in pathologic angiogenesis, including in cancer and tissue ischemia [8]. In healthy pregnancy, PlGF level increases in serum significantly [9]. In the placenta, it is expressed in giant trophoblast cells and decidua natural killer cells [10]. Although it is dispensable for embryogenesis, recent data support its function in fetal cerebrovascular development [11]. Compared with normotensive controls, circulating levels of sFlt-1 is increased, and free VEGF and free PIGF are decreased before the onset of clinical disease in preeclamptic women $[12,13]$. Reducing levels of PlGF serum in the first trimester causes angiogenesis placenta imbalance, which leads to preeclampsia in the second or third trimester [14].

Today, a large number of women are suffering from preeclampsia during pregnancy. Unfortunately, the leading causes of this disease have not yet been known, which it seems that the mutation in the PlGF gene is one of the leading causes of the disease [15].

This study aimed to examine the polymorphisms of the PLGF gene in preeclamptic women compared with healthy pregnancies. Regarding the importance of the issue, we attempted to obtain enough statistical information to consider single nucleotide polymorphisms (SNPs) for identifying individuals predisposed to the disease through identifying possible mutations associated with preeclampsia in affected individuals.

\section{METHODS}

\section{Ethics Statement}

This study was approved by the Ethics committee of Islamic Azad University- Science and research branch (Approval number: IR.I-AU.SRB.REC.1397.111). Study design and patients: This is a case-control study in which a total of 50 women with preeclampsia (approved by medical history results) versus 50 healthy women include pregnant women without a history of hypertension and on average with $110 \mathrm{mmHg}$ systolic blood pressure and 70 diastolic blood pressure (without history of preeclampsia) referring to hospitals in Tehran were examined between the years 2017 to 2018. All patients provided written informed consent.

Exclusion criteria included the history of cardiovascular disease and metabolic disease, history of hypertension before pregnancy, history of smoking and cigarette and chronic hypertension, and kidney disease. Sanger sequencing method was used to determine the genotypes. The amount of $5 \mathrm{ml}$ peripheral blood samples were collected from patients, divided into clotting tubes for serum separation and in other tubes containing EDTA (Ethylenediaminetetraacetic acid) anticoagulant for DNA extraction. Blood samples were stored at a temperature of $-20^{\circ} \mathrm{C}$.

Table 1. The Primers Sequences for the PlGF Gene

\begin{tabular}{ccc}
\hline & Primer Sequences & $\begin{array}{c}\text { Product } \\
\text { length }\end{array}$ \\
\hline Exon1 & 3'CTGGCTAGGAATCCCTTGG5' & 1001bp \\
& 3'TTCCCAAACGAAAGAAGTCC5' & \\
Exon2 & 3'GCAGCAAGTGGGAAGGAACC5' & 614bp \\
& 3'ATGGAAACGCATGGTCAGATC5' \\
Exon3 & 3'TTCCATCCAACAGATGAGC5' & 660 bp \\
& 3'GACTTCATACAAGCAGATTTCT5' \\
Exon6 & 3'ATGAAGCCGGAAAGGTAA5' & 332bp \\
& 3'GCCCTAGAGTCTTGAGGA5' \\
Exon7 & 3'TGAGGGTTAAGAAGACAGTG5' & 1272bp \\
& 3'CGAGGCTTTTGAGATAAGG5' \\
\hline
\end{tabular}

Polymerase chain reaction (PCR) and sequencing: The PCR reaction was performed in $25 \mu$ l volume containing 100-300 ng of extracted DNA, 1X PCR buffer ( $50 \mathrm{mM} \mathrm{KCl} ; 10 \mathrm{mM}$ Tris- $\mathrm{HCl} ; 1.5 \mathrm{mM} \mathrm{MgCl}_{2}$ ), $2 \mathrm{mM} \mathrm{MgCl}_{2}, 200 \mu \mathrm{M}$ dNTP mix, and double-distilled water was added up to $25 \mu \mathrm{l}$ with $1 \mathrm{U}$ of Taq DNA polymerase (super Taq DNA polymerase, Gen Fanavaran co., Tehran, Iran) and $0.4 \mu \mathrm{M}$ of each oligonucleotide primer in Thermocycler (EppendorfNexus-Germany). PCR program was performed as the first denaturation in $95^{\circ} \mathrm{C}$ for $5 \mathrm{~min}$, Denaturation in $95^{\circ} \mathrm{C} 30 \mathrm{~s}$, Annealing in $58^{\circ} \mathrm{C} 30 \mathrm{~s}$, Elongation $72^{\circ} \mathrm{C} 30 \mathrm{~s}$, Final extension $72^{\circ} \mathrm{C} 5 \mathrm{~min}$. PCR products were loaded on $1 \%$ agarose gel followed by ethidium bromide 
staining to confirm the detected, amplified fragments (Primers were summarized in Table 1).

To determine the genotypes of PCR products, samples were sent to the sequence that ultimately the results obtained from the charts of the sequenced examples were examined by Finch TV software, and the accuracy of the work was confirmed.

Statistical analysis: SPSS software version 20 was used for data analysis, and only 5\% was considered as an acceptable rate of the type 1 error. The SHEsis software was used to examine the Hardy-Weinberg equilibrium. Also, given the status of data distribution, Independent samples t-test, Mann-Whitney U, and one-way ANOVA or Kruskal-Wallis were used. Odds ratios (OR) with 95\% confidence intervals were calculated to determine the odds of developing preeclampsia when the individual has the gene variants of interest. Values of $\mathrm{P}<$ 0.05 were considered statistically significant.

\section{RESULTS}

The demographic and clinical characteristics of the subjects are presented in Table 2. The results of this study showed that there was a significant difference between the two groups of patients and control in terms of weight and blood pressure. So that the weight in the patient group was significantly higher than that of the control group $(\mathrm{P}<0.001$, $\mathrm{OR}=2.55)$. As well as, according to this study, it was found that systolic blood pressure $(\mathrm{P}<0.001)$ and diastolic blood pressure $(\mathrm{P}<$ 0.001 ) had a significant difference between the two groups, so that blood pressure in the patient group was higher than the control group, but there was no significant difference in body mass index $(\mathrm{P}=0.1, \mathrm{OR}=$ $0.57)$ and age $(P=0.2, \mathrm{OR}=0.36)$ between both case and control groups (Table 2).

Table 2. Anthropometry and blood pressure data in both patient (case) and control groups

\begin{tabular}{|c|c|c|c|c|}
\hline Characteristics & Case group & Control group & OR (CI 95\%) & Pvalue \\
\hline Age, (years) & $25.8(7.16)$ & $24.7(6.22)$ & 0.36 & 0.2 \\
\hline Gestational age, (weeks) & $32.9(4.02)$ & $33.1(4.71)$ & 0.45 & 0.7 \\
\hline Gestational weight gain, (kg) & $12.7(8.50-16.50)$ & $10.0(6.75-13.55)$ & 2.55 & 0.003 \\
\hline Systolic blood pressure, (mmHg) & $170(160.0-180.0)$ & $110(100.0-120.0)$ & 6.00 & $<0.001$ \\
\hline Diastolic blood pressure, (mmHg) & $110(100.0-120.0)$ & $70(70.0-80.0)$ & 6.05 & $<0.001$ \\
\hline Body mass index, $\left(\mathrm{kg} / \mathrm{m}^{2}\right)$ & $25.05(21.63-28.13)$ & $23.35(20.53-26.90)$ & 0.57 & 0.1 \\
\hline
\end{tabular}

Data presented as mean (SD) or median (ranges)

PCR product bands were determined in Figure 1. Sanger Sequencing data for PLGF gene and comparing it with the reference genome in the Ensemble database indicated that there are four single nucleotide polymorphisms in the case groups of Exon7. These SNPs include rs12411, rs869273721, rs1472556360, rs1169394182. Variants rs869273721 and rs1169394182 have been shown as intronic deletions, $\mathrm{A} /$ - and $\mathrm{C} /$ - respectively, that were without clinical significance and reported only in one and two patients, and these variants were considered rare. Also, rs1472556360 is a T/A SNP found in 3`UTR of exon number 7 and was reported only in one patient and considered rare. Rs12411 was reported in 6 of 25 patients (24\%). Therefore it was considered as a common variant. There were three single nucleotide polymorphisms in the case groups of exon6 include rs1464547123, rs937495410 and rs1048230880. Rs1464547123 is a TT/TA SNP that has been found in the intron of exon number 6 and were reported in 8 of 25 patients (32\%). Therefore it was considered a common variant. There were four single nucleotide polymorphisms in Exon 3 in the case groups. Rs772978877, rs1157624583 and rs756979630 were alternative variants without any clinical significance and rs 1157624583 reported in 6 of 25 patients (24\%). There were four variants in Exon 2 in the case group. Rs772978877, rs1369722917 and rs761111694 as a Shuffle change without any clinical significance have been seen in one of the patients and the variant rs 1399853427 was reported in 4 of 25 cases (16\%) (Tables 3 and 4).

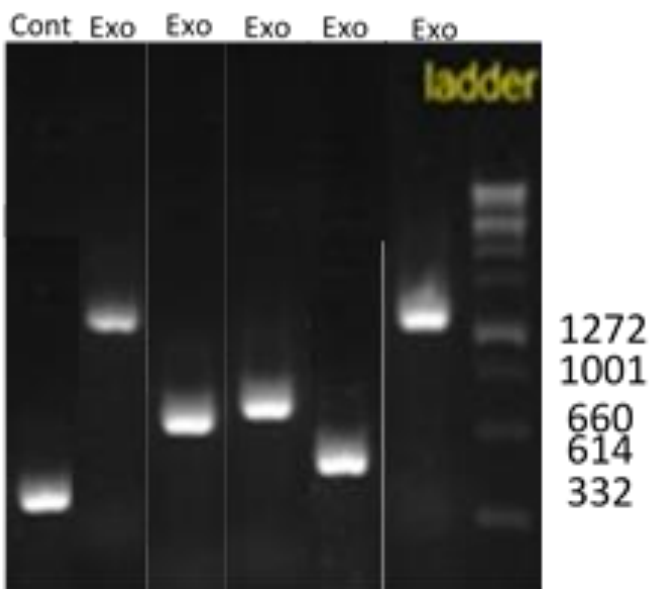

Figure 1. Polymerase chain reaction amplification. Fragments of the gene were detected after electrophoresis on $1 \%$ agarose gel. The Housekeeping gene is GAPDH

\section{DISCUSSION}

Preeclampsia is known to be the third cause of maternal death worldwide. The incidence of preeclampsia has increased probably due to the prevalence of disorders such as hypertension, diabetes, and obesity [16]. Approximately one-tenth of all maternal mortality in Africa, Asia, and a quarter in Latin America is associated with diseases like hypertension during pregnancy. Preeclampsia is prevalent in the first pregnancy. Women with preeclampsia in their early pregnancy are more 
likely to develop preeclampsia in their later pregnancies. Women with high blood pressure, obesity, diabetes, and autoimmune diseases such as lupus, as well as various hereditary thrombophilia such as leukemia Factor V, kidney disease, multiple pregnancies (twin or more), and high maternal age, are a greater risk for preeclampsia. PlGF plays an important role in the angiogenesis of natural tissues and also in tumors, the lack of which is associated with angiogenesis deficiency $[17,18]$.

Table 3. All variants have been seen in exon 2, 3, 6 and 7

\begin{tabular}{|c|c|c|c|}
\hline Variants & Nucleotide change & Mutation site & Abundance, $\mathrm{N}=\mathbf{2 5}$ \\
\hline \multicolumn{4}{|l|}{ Exon 2} \\
\hline rs 1399853427 & $\mathrm{C} / \mathrm{T}$ & Trimming & $4(16)$ \\
\hline rs772978877 & $\mathrm{T} / \mathrm{C}$ & Trimming & $1(4)$ \\
\hline rs1369722917 & $-/ \mathrm{T}$ & Trimming & $1(4)$ \\
\hline rs761111694 & $\mathrm{G} / \mathrm{A}$ & Meaningful variant in intron & $1(4)$ \\
\hline \multicolumn{4}{|l|}{ Exon 3} \\
\hline rs756979630 & $-/ G$ & Trimming & $1(4)$ \\
\hline rs772978877 & $\mathrm{T} / \mathrm{C}$ & Replacement & $1(4)$ \\
\hline rs 1157624583 & $\mathrm{C} / \mathrm{T}$ & Synonym Variant & $1(4)$ \\
\hline rs 1312670436 & $\mathrm{G} / \mathrm{C}$ & Replacement & $6(24)$ \\
\hline \multicolumn{4}{|l|}{ Exon 6} \\
\hline rs1464547123 & $\mathrm{TT} / \mathrm{TC}$ & Trimming & $8(32)$ \\
\hline rs937495410 & $\mathrm{C} /-$ & Deleting introns & $1(4)$ \\
\hline rs 1048230880 & $\mathrm{~A} /-$ & Deleting introns & $1(4)$ \\
\hline \multicolumn{4}{|l|}{ Exon 7} \\
\hline rs12411 & $\mathrm{A} / \mathrm{T}$ & 3 UTR & $6(24)$ \\
\hline rs869273721 & $\mathrm{A} /-$ & Deleting introns & $1(4)$ \\
\hline rs 1472556360 & $\mathrm{~T} / \mathrm{A}$ & 3 UTR & $1(4)$ \\
\hline rs1169394182 & $\mathrm{C} /-$ & Deleting introns & $2(8)$ \\
\hline
\end{tabular}

Data in table are presented as No. (\%).

Table 4. Results of Allele and Genotype Frequency Distribution in the most frequent single nucleotide polymorphism (SNP) in the PlGF gene.

\begin{tabular}{|c|c|c|c|c|}
\hline Allele /Genotype & Case $(\mathrm{N}=25)$ & Control $(\mathrm{N}=25)$ & OR (95 CI) & Pvalue \\
\hline \multicolumn{5}{|l|}{ rs12411, exon 7} \\
\hline A & $6(24)$ & $2(8)$ & $1.46(1.22-1.56)$ & 0.04 \\
\hline $\mathrm{T}$ & $19(76)$ & $23(92)$ & & \\
\hline AA & $4(16)$ & $1(4)$ & $1.26(1.08-2.40)$ & 0.1 \\
\hline AT & $2(8)$ & $1(4)$ & & \\
\hline $\mathrm{TT}$ & $19(76)$ & $23(92)$ & & \\
\hline \multicolumn{5}{|c|}{ rs1464547123, exon6 } \\
\hline $\mathrm{C}$ & $17(68)$ & $5(20)$ & $1.13(1.06-1.56)$ & 0.08 \\
\hline $\mathrm{T}$ & $7(28)$ & $20(80)$ & & \\
\hline $\mathrm{CC}$ & & $3(12)$ & $1.2(1.30-2.40)$ & 0.1 \\
\hline $\mathrm{CT}$ & $1(4)$ & $2(8)$ & & \\
\hline $\mathrm{TT}$ & $17(68)$ & $20(80)$ & & \\
\hline \multicolumn{5}{|c|}{ rs1312670436, Exon3 } \\
\hline G & $6(24)$ & $2(8)$ & $1.22(1.08-1.56)$ & 0.8 \\
\hline $\mathrm{C}$ & $19(76)$ & $23(92)$ & & \\
\hline GG & $3(12)$ & $2(8)$ & $1.31(1.12-1.64)$ & 0.1 \\
\hline GC & $3(12)$ & $0(0)$ & & \\
\hline $\mathrm{CC}$ & $19(76)$ & $23(92)$ & & \\
\hline \multicolumn{5}{|c|}{ Rs1399853427, Exon2 } \\
\hline $\mathrm{C}$ & $4(16)$ & $1(4)$ & $1.09(1.01-1.36)$ & 0.2 \\
\hline $\mathrm{T}$ & $21(84)$ & $24(96)$ & & \\
\hline $\mathrm{CC}$ & $3(12)$ & $1(4)$ & $1.01(1.00-1.19)$ & 0.3 \\
\hline $\mathrm{CT}$ & $1(4)$ & $0(0)$ & & \\
\hline $\mathrm{TT}$ & $21(84)$ & $24(96)$ & & \\
\hline
\end{tabular}

PlGF involved in cardiovascular disease, infectious diseases and increases the internal thickness of atherosclerosis. Several studies have shown the critical role of PlGF in pathologic conditions such as ischemia, tumor formation, cardiovascular disease, and inflammatory processes [19]. In one study, changes in plasma PIGF were assessed in two groups. Results showed a strong association between elevated PlGF with age and gender. Then, the relationship between single nucleotide polymorphisms (SNPs) in PlGF and its plasma levels was investigated. Two polymorphisms rs11850328 and rs2268614 showed a significant relation with PlGF plasma levels.

The present study aimed to investigate the exons of the PIGF gene in women with preeclampsia. The results of our research in exon 7, variants rs869273721 and rs1169394182 have been shown an intronic deletion in one and two patients, respectively, and the variant is considered rare without clinical importance. Also, the rs1472556360 option was seen as a single nucleotide replacement in one patient, which was reported as a rare variant. In exon six have been shown three single- 
nucleotide variants that rs937495410 and rs1048230880 variants were rarely reported and singlenucleotide polymorphism rs 1464547123 was more common in both control and control groups.

In a similar study, Vodolazkaia et al. examined the effects of single nucleotide polymorphisms in the angiogenesis and its association with endometriosis. These genes included VEGF, PLGF, VEGFR1, and HIF-1a. The results of this study showed that there was a significant relationship between rs 2268613 polymorphism in the PLGF gene and endometriosis. Women with AA genotype had a lower level of their blood PLGF than those with AG genotype [20].

In the present study, rs12411 have been reported in exon 7 in 6 of 25 patients and 2 healthy subjects. Sorice $\mathrm{R}$ et al. examined a total of 1812 European female patients with PLGF gene polymorphisms in a cohort study. The five SNPs located in the PGF gene region were: the rs12411, rs8185 and rs2268613 polymorphisms placed in the 39 regulative region; the rs2268614 variant located in intron 3 and the rs11850328 variant located in the region upstream the PGF gene. The results of this study showed that three polymorphisms rs12411, rs11850328 and rs2268614 were significant in the studied population [21].

Conclusion: This study aimed to investigate the PLGF gene in women with preeclampsia. Four common variants rs12411, rs1464547123, rs1399853427 and rs 1312670436 were reported in these patients. According to the statistical results, it is necessary to use the number of more people in this test to establish a meaningful relationship between preeclamptic patients. It is suggested that the variants of the present study be repeated with a larger sample size in Iranian women to get better results.

\section{Acknowledgments}

This study was financially supported by Tehran central Azad University to the masters and administrative staff of Baghiatalah Hospital and M. Nikzad.

\section{Conflict of Interest}

There is no conflict of interest in this study.

\section{REFERENCES}

1. Chaiworapongsa T, Chaemsaithong P, Yeo L, Romero R. Preeclampsia part 1: current understanding of its pathophysiology. Nat Rev Nephrol. 2014;10(8):466-80. doi: 10.1038/nrneph.2014.102 pmid: 25003615

2. Girardi G. Pravastatin for Preeclampsia Prevention and Treatment. Preeclampsia. Comprehensive Gynecology and Obstetrics2018. p. 239-51

3. Ghulmiyyah L, Sibai B. Maternal mortality from preeclampsia/eclampsia. Semin Perinatol. 2012;36(1):56-9. doi: 10.1053/j.semperi.2011.09.011 pmid: 22280867

4. Lisonkova S, Joseph KS. Incidence of preeclampsia: risk factors and outcomes associated with early- versus late-onset disease. Am J Obstet Gynecol. 2013;209(6):544 e1- e12. doi: 10.1016/j.ajog.2013.08.019 pmid: 23973398

5. Rojas JC, Luna M, Rangel-Nava H, Banos D, Collados MT. [Genetic thrombophilia and markers of endothelial activation in patients with preeclampsia]. Ginecol Obstet Mex. 2010;78(8):401-9. pmid: 20939248

6. Valenzuela FJ, Perez-Sepulveda A, Torres MJ, Correa P, Repetto GM, Illanes SE. Pathogenesis of preeclampsia: the genetic component. J Pregnancy. 2012;2012:632732. doi: 10.1155/2012/632732 pmid: 22175024

7. Furuya M, Kurasawa K, Nagahama K, Kawachi K, Nozawa A, Takahashi $\mathrm{T}$, et al. Disrupted balance of angiogenic and antiangiogenic signalings in preeclampsia. J Pregnancy. 2011;2011:123717. doi: 10.1155/2011/123717 pmid: 21490787

8. Dewerchin M, Carmeliet P. PlGF: a multitasking cytokine with disease-restricted activity. Cold Spring Harb Perspect Med. 2012;2(8). doi: 10.1101/cshperspect.a011056 pmid: 22908198

9. Carmeliet P, Moons L, Luttun A, Vincenti V, Compernolle V, De Mol M, et al. Synergism between vascular endothelial growth factor and placental growth factor contributes to angiogenesis and plasma extravasation in pathological conditions. Nat Med. 2001;7(5):575-83. doi: 10.1038/87904 pmid: 11329059

10. Aasa KL, Zavan B, Luna RL, Wong PG, Ventura NM, Tse MY, et al. Placental growth factor influences maternal cardiovascular adaptation to pregnancy in mice. Biol Reprod. 2015;92(2):44. doi: 10.1095/biolreprod.114.124677 pmid: 25537372

11. Luna RL, Kay VR, Ratsep MT, Khalaj K, Bidarimath M, Peterson N, et al. Placental growth factor deficiency is associated with impaired cerebral vascular development in mice. Mol Hum Reprod. 2016;22(2):130-42. doi: 10.1093/molehr/gav069 pmid: 26646502

12. Lam C, Lim KH, Karumanchi SA. Circulating angiogenic factors in the pathogenesis and prediction of preeclampsia. Hypertension. 2005;46(5):1077-85. doi 10.1161/01.HYP.0000187899.34379.b0 pmid: 16230516

13. Haram K, Mortensen JH, Nagy B. Genetic aspects of preeclampsia and the HELLP syndrome. J Pregnancy. 2014;2014:910751. doi: 10.1155/2014/910751 pmid: 24991435

14. Kurtoglu E, Avci B, Kokcu A, Celik H, Cengiz Dura M, Malatyalioglu E, et al. Serum VEGF and PGF may be significant markers in prediction of severity of preeclampsia. $\mathrm{J}$ Matern Fetal Neonatal Med. 2016;29(12):1987-92. doi: 10.3109/14767058.2015.1072157 pmid: 26333278

15. Denney JM, Bird C, Gendron-Fitzpatrick A, Sampene E, Bird IM, Shah DM. Renin-angiotensin system transgenic mouse model recapitulates pathophysiology similar to human preeclampsia with renal injury that may be mediated through VEGF. Am J Physiol Renal Physiol. 2017;312(3):F445-F55. doi: 10.1152 /ajprenal.00108.2016 pmid: 27927648

16. Jeyabalan A. Epidemiology of preeclampsia: impact of obesity. Nutr Rev. 2013;71 Suppl 1:S18-25. doi: 10.1111/nure.12055 pmid: 24147919

17. Mongraw-Chaffin ML, Cirillo PM, Cohn BA. Preeclampsia and cardiovascular disease death: prospective evidence from the child health and development studies cohort. Hypertension. 2010;56(1):166-71. doi: 10.1161/HYPERTENSIONAHA.110.150078 pmid: 20516394

18. Shamsi U, Saleem S, Nishter N, Ameen A. Epidemiology and risk factors of preeclampsia; an overview of observational studies. Al Ameen J Med Sci. 2013;6(4):292-300.

19. Davis EF, Lazdam M, Lewandowski AJ, Worton SA, Kelly B, Kenworthy $\mathrm{Y}$, et al. Cardiovascular risk factors in children and young adults born to preeclamptic pregnancies: a systematic review. Pediatrics. 2012;129(6):e1552-61. doi: 10.1542/peds.2011-3093 pmid: 22614768

20. Vodolazkaia A, Yesilyurt BT, Kyama CM, Bokor A, Schols D, Huskens D, et al. Vascular endothelial growth factor pathway in endometriosis: genetic variants and plasma biomarkers. Fertil Steril. 2016;105(4):988-96. doi: 10.1016/j.fertnstert.2015.12.016 pmid: 26773192 
21. Sorice R, Ruggiero D, Nutile T, Aversano M, Husemoen L, Linneberg A, et al. Genetic and environmental factors influencing the Placental Growth Factor (PGF) variation in two populations. PLoS One. 2012;7(8):e42537. doi: 10.1371/journal.pone. 0042537 pmid: 22916133 